\title{
Farmacogenética, tabaco, alcohol y su efecto sobre el riesgo de desarrollar cáncer
}

\author{
Pharmacogenetics, tobacco, alcohol and its effect on the risk development cancer
}

\author{
Ángela Roco $\mathrm{PhD}^{\mathrm{a}, \mathrm{b}, \mathrm{c}}$, Berta Cerda $\mathrm{MD}^{\mathrm{d}}$, Juan Pablo Cayún $\mathrm{PhD}(\mathrm{c})^{\mathrm{a}}$, \\ Alejandra Lavanderos $\mathrm{PhD}(\mathrm{c})^{\mathrm{a}}$, Juan Carlos Rubilar MSc ${ }^{\mathrm{a}}$, Roberto Cerro MSc ${ }^{\mathrm{a}}$, \\ Christian Acevedo $\mathrm{MD}^{\mathrm{a}}$, Dante Cáceres $\mathrm{PhD}^{\mathrm{a}}$, Nelson Varela ${ }^{\mathrm{a}} \mathrm{PhD}$, Luis A. Quiñones ${ }^{\mathrm{a}} \mathrm{PhD}$
}

\author{
aLaboratorio de Carcinogénesis Química y Farmacogenética, Departamento de Oncología Básico Clínica. Facultad de Medicina, \\ Universidad de Chile, Santiago, Chile \\ bEscuela de Bioquímica, Facultad de Ciencias de la Vida, Universidad Andrés Bello, Santiago, Chile \\ 'Servicio de Salud Metropolitano Occidente, Ministerio de Salud, Chile \\ Instituto Nacional del Cáncer, SSM Norte, Ministerio de Salud, Chile
}

Recibido el 22-05-2018; aceptado el 7-06-2018

\section{Resumen}

El cáncer es la segunda causa de muerte en el mundo, según datos de la Organización Mundial de la Salud (OMS) en el año 2015 ocasionó 8,8 millones de muertes. Dentro de los factores de riesgo para el desarrollo de cáncer se encuentran el tabaquismo y el consumo de alcohol. En Chile el 33,6\% de la población fuma y un 21,2 \% de los jóvenes. El consumo de alcohol en la población chilena es de 74,5 $\%$ y en los jóvenes de un 12,2 \%. Entre los factores fisiológicos que influyen en el desarrollo de cáncer, el factor genético juega un rol relevante, habiéndose demostrado que la presencia de polimorfismos genéticos alteran la capacidad del organismo de eliminar contaminantes y aumentan el riesgo de desarrollar cáncer. Lo mismo ocurre con polimorfismos que impiden la reparación de ADN debido a daños producidos por efecto de contaminantes ambientales como el humo de cigarrillo. El objetivo de esta revisión es analizar el estado del arte de la relación entre farmacogenética, tabaco y alcohol como factores de riesgo para el desarrollo de cáncer. Los resultados sugieren que la presencia de polimorfismos que alteran la función de enzimas de biotransformación fase I (CYP1A1, CYP1E1) y fase II (GST), además de polimorfismos en enzimas de reparación del ADN (ERCC1/ERCC2) aumentan el riesgo de cáncer inducido por el hábito tabáquico y alcohólico. Esta asociación es importante, si consideramos que en la población chilena el hábito de fumar y beber alcohol es altamente prevalente.
Palabras clave: Farmacogenética; cáncer; tabaco; alcohol 


\begin{abstract}
Cancer is the second leading cause of death in the world, causing 8.8 million deaths in 2015 according to the World Health Organization (WHO). Risk factors for cancer include smoking and alcohol consumption. In Chile, $33.6 \%$ of the population and $21.2 \%$ of young people smokes. Alcohol consumption in the Chilean population is $74.5 \%$ and $12.2 \%$ in young people. Among the physiological factors that influence the development of cancer, the genetic factor plays a relevant role. It has been shown that the presence of genetic polymorphisms that alter the ability of the body to eliminate contaminants increase the risk of developing cancer. The same applies to polymorphisms that prevent DNA repair due to damage caused by environmental pollutants such as cigarette smoke. The objective of this review is to analyze the state of the art of the relationship between pharmacogenetics, smoking, and alcohol consumption as risk factors for the development of cancer. In conclusion, the results suggest that the presence of polymorphisms that alter the function of biotransformation enzymes phase I (CYP1A1, CYP1E1) and phase II (GST), as well as polymorphisms in DNA repair enzymes (ERCC1 / ERCC2), increase the risk of cancer induced by smoking and alcohol consumption. This association is important considering that smoking and drinking alcohol are highly prevalent among the Chilean population.
\end{abstract}

Keywords:

pharmacogenetics; cancer;

tobacco;

alcohol

\section{Introducción}

El cáncer es la segunda causa de muerte en el mundo, en el año 2015 según datos de la Organización Mundial de la Salud (OMS) ocasionó 8,8 millones de defunciones. Cerca del 70\% de las muertes por cáncer se registran en países de ingresos medios y bajos. El impacto económico del cáncer es enorme y va en aumento, el costo estimado atribuible a la enfermedad en el año 2010 ascendió a US $\$ 1,16$ billones ${ }^{1}$. El término "cáncer infantil" se utiliza para designar distintos tipos de cáncer que pueden aparecer en los niños antes de cumplir los 15 años. El cáncer infantil es poco frecuente, pues representa entre un $0,5 \%$ y un $4,6 \%$ de la carga total de morbilidad por esta causa. Las tasas mundiales de incidencia oscilan entre 50 y 200 por cada millón de niños en las distintas partes del planeta ${ }^{2}$.

Alrededor de un tercio de las muertes por cáncer se debe a los cinco principales factores de riesgo conductuales y dietéticos: índice de masa corporal elevado, ingesta reducida de frutas y verduras, falta de actividad física, consumo de tabaco y consumo de alcohol. El tabaquismo es el principal factor de riesgo y ocasiona aproximadamente el $22 \%$ de las muertes por cáncer en el mundo ${ }^{1}$.

Según las estimaciones realizadas en base a la información provenientes de los registros poblacionales de cáncer y de la mortalidad entre 2003 y 2007 en Chile, la tasa de incidencia anual de cáncer fue de 216,9 por 100 mil habitantes, lo que equivale a aproximadamente 35.000 casos nuevos al año ${ }^{3}$. En los hombres, los canceres más incidentes son próstata, estómago, piel no melanoma, pulmón, colon y vesícula biliar; en las mujeres los primeros lugares son mama, piel no melanoma, vesícula biliar, cérvico uterino, estómago y colon. En relación con la presentación geográfica, se observa que en el norte del país las tasas más altas corresponden a cánceres del sistema respiratorio, piel y vejiga, mientras que en el sur predominan los cánceres del aparato digestivo (estomago, vesícula biliar y colon).

La incidencia de cáncer en menores de 15 años en Chile es de 12,8 por 100.000 , algo más frecuente en niños $(13,9$ por 100.000$)$ que en niñas ( 11,6 por 100.000$)$, lo que equivale a cerca de 500 nuevos casos al año. El $40,1 \%$ de los casos corresponde a leucemias, el 20,3\% a sistema nervioso central y el $12,7 \%$ a Linfomas ${ }^{3}$. Los subgrupos de cáncer con mayores tasas son la Leucemia linfática $(3,9$ por 1000,000$)$ y la Leucemia mieloide aguda $(0,9 \text { por } 100.000)^{3}$.

Las tasas de mortalidad del año 2013 en Chile para las primeras cinco causas de muerte por tipo de cáncer y las del Mundo correspondientes al año 2012 se muestran en la Tabla $1^{4,5}$. En Chile la primera causa de muerte en ambos sexos en el cáncer de estómago, seguido del cáncer de tráquea, bronquios y pulmón, en tercer lugar se ubica el cáncer de próstata, en hombres la primera causa de muerte es el cáncer de estómago y en mujeres de mama ${ }^{4}$. En el mundo la primera causa de muerte en ambos sexos y en hombres es el cáncer de pulmón y para mujeres el cáncer de mama ${ }^{5}$.

El objetivo de esta revisión es analizar el estado del arte de la relación entre farmacogenética, tabaco, alcohol como factores de riesgo para el desarrollo de cáncer.

\section{Consumo de tabaco y alcohol en población chilena}

\section{Tabaco}

En Chile, según el Estudio Nacional de Drogas en Población Escolar 2015 la edad promedio de inicio de 
Tabla 1. Tasa de Tumores Malignos según Sexo, Chile 2013 y Mundo 2012,5

\begin{tabular}{llll}
\hline Chile & $2013^{*}$ & Mundo & $2012^{*}$ \\
\hline Tumor maligno de & Ambos sexos & Tumor maligno de & Ambos sexos \\
Estómago & 18,4 & Pulmón & 19,7 \\
Tráquea, de los bronquios y del pulmón & 17,2 & Mama & 12,9 \\
Próstata & 11,6 & Hígado & 9,46 \\
Colon & 9,3 & Estómago & 8,9 \\
Mama & 8,0 & Colon & 8,3 \\
Tumor maligno de & Hombres & Tumor maligno de & Hombres \\
Estómago & 24,4 & Pulmón & 20,95 \\
Próstata & 23,4 & Hígado & 14,25 \\
Tráquea, de los bronquios y del pulmón & 20,7 & Estómago & 12,74 \\
Colon & 8,7 & Colon & 10 \\
Hígado & 6,7 & Próstata & 7,8 \\
Tumor maligno de & Mujeres & Tumor maligno de & Mujeres \\
Mama & 15,6 & Mama & 12,9 \\
Tráquea, de los bronquios y del pulmón & 13,8 & Pulmón & 11,05 \\
Estómago & 12,4 & Colon & 6,94 \\
Vesícula biliar & 11,0 & Cervicouterino & 6,82 \\
Colon & 9,8 & Estómago & 5,65 \\
\hline *Tasa por 100 mil habitantes. & &
\end{tabular}

consumo de tabaco es 13,7 años, sin diferencias entre hombres y mujeres. El 66\% de los fumadores señala haber probado tabaco por primera vez antes de los 15 años. La prevalencia de fumar en escolares es de un $21,2 \%$, con un 5,5\% entre 15 a 18 años y un $16,5 \%$ entre 19 a 25 años, alcanzando un máximo en los grupos de 26 a 34 y 34 a 44 años ${ }^{6}$.

Por otra parte, en la Encuesta Nacional de Salud de Chile desarrollada en el año 2016-2017 por el Ministerio de Salud ${ }^{7}$, se evidenció la magnitud de las Enfermedades Crónicas No Transmisibles (ECNT) en términos de morbilidad, al medir por tercera vez su prevalencia y la de sus factores de riesgo a nivel nacional. Los resultados muestran un predominio de estilos de vida poco saludables, con elevado consumo de tabaco $(33,6 \%)$, sedentarismo $(86,7 \%)$, consumo riesgoso de alcohol $(11,7 \%)$, sobrepeso $(39,8 \%)$ y obesidad $(3,2 \%)$, entre otros. La alta prevalencia de hábitos de vida poco saludables queda en evidencia también entre los más jóvenes. En la Encuesta Mundial de Tabaquismo en Jóvenes (GITS de sus siglas en inglés) 2008-2010 se observa un consumo de cualquier producto de tabaco de un 35,1\% y un consumo de Cigarrillos en el último mes de 34,2\%, en los jóvenes encuestados de la Región Metropolitana ${ }^{8}$. Otro dato interesante de la ENS 20162017 es que el 15,2\% está expuesto al humo de tabaco en el hogar y un 20,3\% está expuesto en el lugar de estudio o trabajo ${ }^{7}$.

Se ha postulado que la reducción del consumo de tabaco impactaría en la incidencia de enfermedades cardiovasculares y respiratorias en primer lugar, y posteriormente en cáncer y otras enfermedades ${ }^{9,10}$. Se- gún el estudio de Carga de Enfermedad atribuible al Tabaquismo en Chile del año 2014, se concluye que el $87 \%$ de las muertes por cáncer de pulmón y un 32\% del resto de los cánceres son atribuibles al consumo de tabaco $^{11}$. Este estudio muestra que el 73\% cáncer esófago, 85\% cáncer de laringe, 47\% cáncer vejiga, 27\% cáncer de estómago y un 21\% leucemias son atribuible al tabaquismo en Chile ${ }^{11}$. Varios estudios han mostrado el impacto de fumar o vivir en ambientes con humo de tabaco y el mayor riesgo de desarrollar cáncer ${ }^{12,13,14}$.

\section{Alcohol}

Basados en estimaciones de la OMS, en 2010, el consumo total de alcohol per cápita en todo el mundo registró un promedio de 21,2 litros de alcohol puro entre los hombres, y 8,9 litros entre las mujeres ${ }^{15}$. El consumo de alcohol es uno de los más importantes factores de riesgo para el desarrollo de cáncer y uno de sus factores más evitables ${ }^{15}$. En Chile, el alcohol es el principal factor de carga atribuible, responsable del $12,4 \%$ de los años de vida perdidos por muerte o discapacidad ${ }^{16}$. La encuesta nacional de salud (ENS) 20092010 muestra en relación con el hábito alcohólico de la población chilena, que un 74,5\% de la población ha consumido alcohol en el último año, un 57,6\% en el último mes y un $36,1 \%$ en la última semana ${ }^{17}$. La ENS 2016-2017 sólo evalúa el consumo riesgoso de alcohol que se presenta en el $11,7 \%$ de la población, con un $20,5 \%$ en hombres y un 3,3\% en mujeres ${ }^{7}$. El mayor consumo riesgoso se presenta en los rangos etarios 15 a 19 años $(12,2 \%)$ y entre 20 a 29 años $(18,8 \%)^{7}$. Según el duodécimo estudio de drogas en población general 
chilena del año 2016, los resultados para uso de alcohol en la población general muestran que la prevalencia de "consumo alguna vez en la vida" varía de $79,1 \%$ en 2014 a 79,9\% en 2016 y el "consumo en el último mes" baja de $48,9 \%$ a $46,0 \%$, estas diferencias no son estadísticamente significativas. Por otra parte, las mayores prevalencias de consumo de alcohol se presentan en los rangos etarios de 19 a 25 años $(56,7 \%)$ y en el rango de 26 a 34 años $(58,1 \%)^{18}$.

En general, la cantidad de alcohol consumido en el tiempo, y no el tipo de bebida alcohólica, parece ser el factor más importante en el riesgo de desarrollar cáncer. Unos de los efectos del alcohol es una disminución en la absorción de folato, lo que se ha correlacionado con mayor riesgo de cáncer de mama y colorectal ${ }^{19,20}$. Se ha descrito en varias publicaciones la asociación entre mayor riesgo por consumo de alcohol en diferentes tipos de cáncer ${ }^{21,22}$, entre ellos el estudio de Fedirko y colaboradores que asocia un aumento del riesgo de cáncer colorectal con el consumo de 12,5 g/día de etanol $^{23}$. Plichart y su colaboradores demostraron que el consumo paterno de tabaco (mayor a 20 cigarrillos/ día) y el consumos materno de bebidas con cafeína (mayor a tres tazas de café al día o de una taza de té al día) durante el embarazo se correlaciona con un mayor riesgo de desarrollar tumores malignos del sistema nervioso central de la infancia ${ }^{24}$.

\section{Metabolismo hidrocarburos aromáticos presentes en el humo del cigarrillo y farmacogenética}

Los hidrocarburos aromáticos policíclicos (HAP) como los que se encuentran en el humo del cigarrillo (benzo(a)pireno), y en el medio ambiente en general, presentan elevados niveles en Santiago de Chile y en otras ciudades del mundo produciendo dramáticos cambios en el metabolismo de las monooxigenasas del sistema Citocromo P450 (CYP). Estos compuestos son eliminados del organismo a través de eficaces sistemas de desintoxicación, especialmente por la enzima de fase I monooxigenasa CYP1A1, cuyas reacciones, además de estar involucradas en el metabolismo de fármacos, síntesis de colesterol, esteroides y otros lípidos, están involucradas en la bioactivación de un gran número de compuestos pre-carcinógenos ${ }^{25,26}$, entre ellos los contaminantes ambientales presentes en el humo del cigarrillo ${ }^{27,28,29}$.

Los compuestos pro-carcinógenos al ser activados se transforman en compuestos carcinogénicos, los cuales se intercalan en el ADN produciendo alteraciones, si es que la maquinaria de reparación de $\mathrm{ADN}$ no es capaz de eliminarlas (Figura 1). La enzima CYP1A1 metaboliza benzo(a)pireno a un epóxido, el cual es hi- drolizado por la epóxido hidrolasa a un dihidrodiol, nuevamente actúa la enzima CYP1A1 que transforma este dihidrodiol a una especie altamente reactiva: diolepóxido [benzo(a)pireno-7,8-dihidrodiol-9,10-epóxido] que se une covalentemente al ADN, produciendo aductos con guanina ${ }^{25}$. El metabolito diol-epóxido puede ser desintoxicado por la familia de enzimas de biotransformación Glutatión S-transferasas (GSTs), en particular por GSTM1 y GSTP1 (Figura 1) ${ }^{25}$.

El gen CYP1A1 presenta polimorfismos, de ellos los dos más secuenciados y estudiados $C Y P 1 A 1^{\star} 2 A$ y $C Y P 1 A 1^{*} 2 C$ que aumentan la actividad enzimáti$\mathrm{ca}^{29,30,31}$. La principal función de las enzimas Glutatión $\mathrm{S}$-Transferasas es detoxicar fármacos, compuestos contaminantes, carcinógenos y mutágenos por conjugación con glutatión (GSH), se han descrito las mutantes null para GSTM1 y GSTT1 donde no hay enzima y la mutante de GSTP1 (rs1695) que disminuye la actividad enzimática (metabolizador pobre) 29,30,31.

Los sistemas de reparación del ADN determinan, en parte, el grado de daño ocasionado por la exposición a agentes ambientales con potencial cancerígeno. Esto es debido a que estos mecanismos de reparación forman la "segunda línea" de defensa frente a posibles efectos tóxicos, complementando así los mecanismos de metabolización. Por tanto, la respuesta individual al daño en el ADN inducido por agentes xenobióticos está también condicionada por la eficacia de los sistemas de reparación. El sistema de reparación por NER (Nucleotide Excision Repair) es un grupo de proteínas que son capaces de reparar el daño en el ADN producido por la formación de aductos por contaminantes ambientales (por ejemplo: humo del cigarrillo) (Figura 1) ${ }^{32}$. Los polimorfismos genéticos de las enzimas implicadas en estos procesos de reparación van a determinar su capacidad funcional, pudiendo conducir a una menor capacidad de reparación del $\mathrm{ADN}$, que puede ser la base de una tasa de mutación más alta, y como consecuencia última provocar un aumento de riesgo de desarrollo de procesos tumorales ${ }^{33-35}$. Los polimorfismos en ERCC1 y $E R C C 2$ han sido asociados a un mayor riesgo para el desarrollo de cáncer de piel, cáncer de mama y cáncer colorectal $^{36-40}$.

\section{Metabolismo alcohol y farmacogenética}

En la ruta de metabolización el etanol se oxida a acetaldehído mediante las enzimas alcohol deshidrogenasa $(\mathrm{ADH})$, que son codificadas por los genes $A D$ H1B y $A D H 1 C$, a través de la enzima citocromo P450 2E1 (CYP2E1) y por microorganismos que habitan en el tracto gastrointestinal. El acetaldehído, el metabolito más tóxico del etanol, se considera un agente can- 


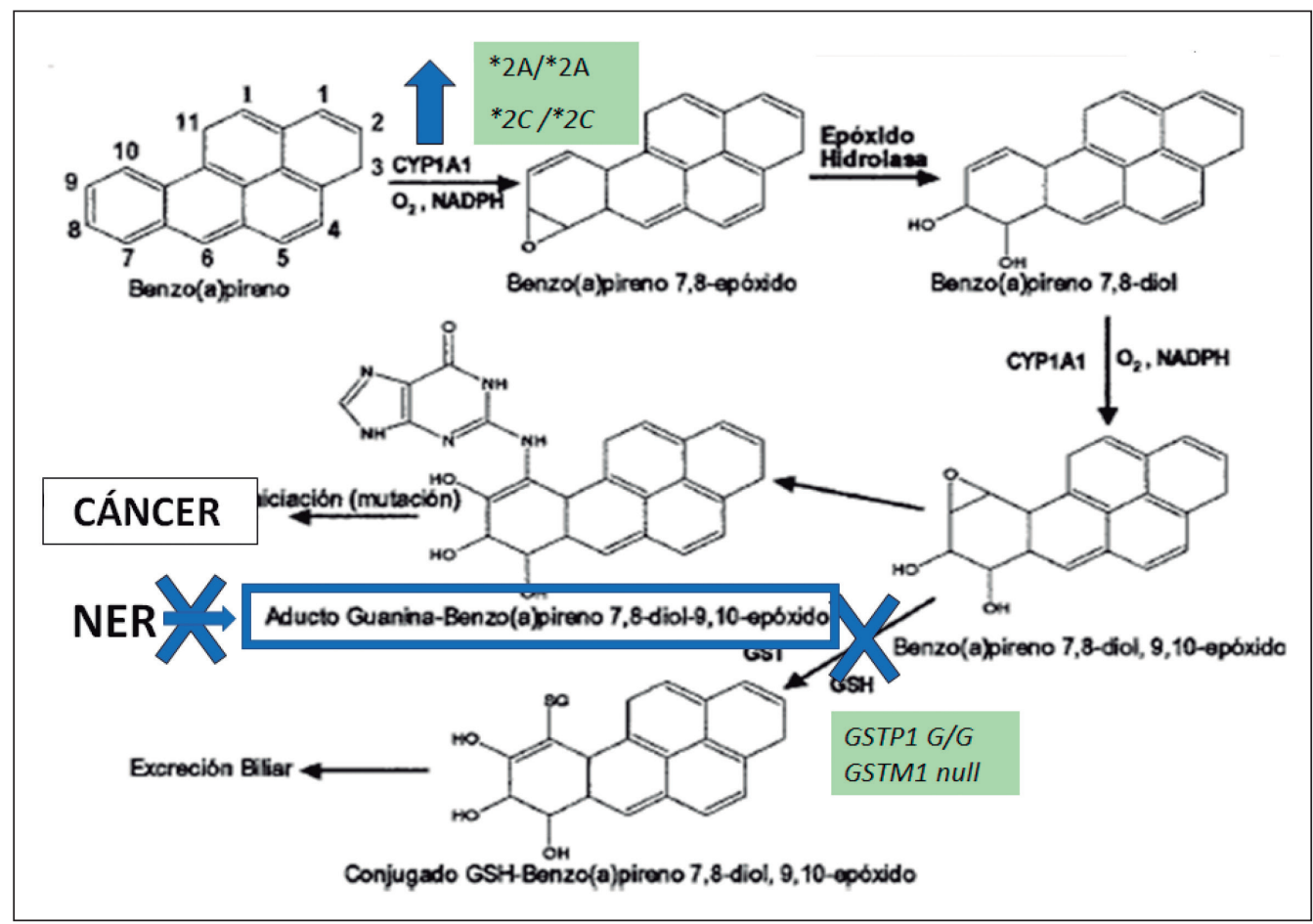

Figura 1. Metabolismo benzo(a)pireno por CYP1A1 y GST ${ }^{23}$. Polimorfismos CYP1A1 *2A/*2A y *2C/*2C aumentan activada enzimática y su efecto es aumentar producción del intermediario Benzo(a)pireno 7,8 diol,9,10 -epóxido. Polimorfismos GSTP1 G/G o mutante GSTM1 null no permiten conjugar con glutación el intermediario Benzo(a)pireno 7,8 diol,9,10 -epóxido, la única opción de este intermediario reactivo es formar aductos con Guanina. Si existen mutaciones en el Sistema de Reparación de ADN (NER, de sus siglas en inglés) se generará el inicio del proceso tumoral.

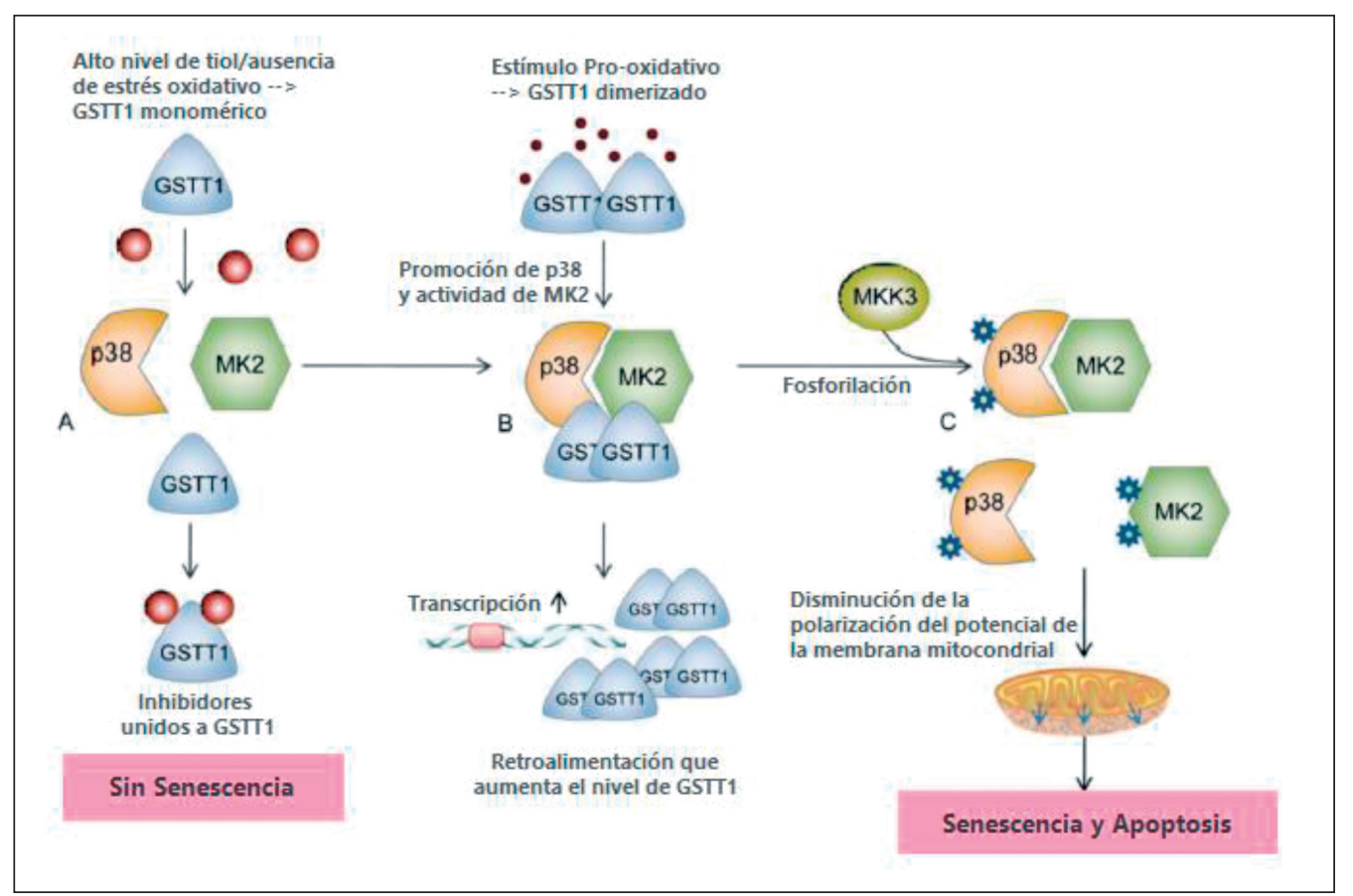

Figura 2. Esquema de los roles potenciales de GSTT1 en la prevención de la tumorigénesis en las células. (A) GSTT1 monomérico se disocia de p38 y MK2 y se une a su inhibidor. (B) En presencia de estímulos pro-oxidativos, GSTT1 se dimeriza y se une a p38 y MK2, aumentado su expresión. (C) p38 y MK2 fosforilados interactúan con MKK3 e inician la reducción de la polarización de la membrana mitocondrial, activando apoptosis y senescencia. Abreviaturas: GSTT1, glutatión S-transferasa theta 1; MK2: proteína quinasa 2 activada por mitógeno; MKK3: proteína quinasa 3 activada por mitógeno (Adaptada de Wang, Y., et al. 2016) ${ }^{44}$. 
Tabla 2. Frecuencia alélica de variantes genéticas (polimorfismos) de enzimas de fase I y fase II en diferentes etnias ${ }^{45,49,50}$

\begin{tabular}{|c|c|c|c|c|c|}
\hline Gen & Polimorfismo & Americanos & Este asiático & Europeos & Chile \\
\hline \multirow[t]{2}{*}{ CYP1A1 } & rs4646903 $(* 2 A)$ & 0,411 & 0,430 & 0,130 & 0,37 \\
\hline & rs1048943 (*2C) & 0,354 & 0,252 & 0,035 & 0,32 \\
\hline CYP2E1 & rs6413432 *6 & 0,173 & 0,272 & 0,105 & 0,22 \\
\hline GSTM1 & null & sd & 0,290 & 0,050 & 0,22 \\
\hline GSTT1 & null & sd & 0,200 & 0,520 & 0,11 \\
\hline GSTP1 & rs1695 & 0,476 & 0,179 & 0,331 & 0,36 \\
\hline ERCC1 & rs3212986 & 0,354 & 0,299 & 0,25 & 0,37 \\
\hline \multirow[t]{3}{*}{ ERCC2 } & rs13181 & 0,215 & 0,076 & 0,364 & 0,24 \\
\hline & rs1799793 & 0,199 & 0,050 & 0,359 & 0,20 \\
\hline & rs238406 & 0,487 & 0,490 & 0,550 & 0,58 \\
\hline
\end{tabular}

cerígeno, ya que, promueve el desarrollo del cáncer a través de múltiples mecanismos, en los que se incluye la interferencia con la replicación, inducción de daño y formación de aductos de $\mathrm{ADN}^{41}$. Se ha demostrado que el acetaldehído causa alteraciones en el material genético, como mutaciones puntuales, así como también alteraciones cromosómicas macroscópicas ${ }^{42}$. El acetaldehído perjudica el proceso de reparación al ADN, inhibiendo enzimas involucradas ${ }^{43}$. El acetaldehído se oxida a acetato por la enzima aldehído deshidrogenasa 2 (ALDH2). Las sustancias inductoras de cáncer son generadas durante las diversas rutas del metabolismo del alcohol. Entre estas se incluyen el acetaldehído, especies reactivas de oxígeno (ROS) generado por CY$\mathrm{P} 2 \mathrm{E} 1 \mathrm{y}$ aductos formados por las interacciones del acetaldehído o ROS con el ADN ${ }^{44,43}$. Las especies reactivas de oxígeno son metabolizadas por las GST y reducidas por la conjugación de $\mathrm{GSH}^{44}$.

GSTT1 puede impedir la proliferación de células tumorales mediante la inducción de apoptosis y senescencia mediada por p38/MAPKAP quinasa 2 (MK2). Se ha observado que en presencia de niveles incrementados de tiol y en ausencia de estrés oxidativo GSTT1 se encuentra en forma monomérica, frente a la presencia de estímulos pro-oxidativos, GSTT1 forma dímeros que se unen a p38 y MK2 para facilitar la activación de estas quinasas, que a su vez elevan la expresión de GSTT1. Además, GSTT1 interactúa con la proteína quinasa 3 activada por mitógeno (MKK3), p38 fosforilado y MK2, activando la apoptosis y la senescencia (Figura 2). En ausencia de GSTT1 (GSTT1 nulo) no hay enzima y, por lo tanto, no se pueden activar los procesos de apoptosis y senescencia en las células tumorales $^{44}$. La variante GSTT1 nula provoca que aumenten los xenobióticos y los niveles de ROS, generando productos tóxicos y carcinogénicos; principalmente radicales libres que provienen de grupos hidroxilos y superóxidos. Estos productos están asociados a la formación de aductos en el ADN, y de esa manera se inicia un proceso tumoral ${ }^{44}$.

\section{Polimorfismos genéticos relacionados con cáncer}

En la Tabla 2 se muestran las frecuencias de los polimorfismos relacionados con la desintoxicación de hidrocarburos aromáticos presentes en el humo del cigarrillo y con el metabolismo del alcohol asociados a mayor riesgo de cáncer y su frecuencia en diferentes etnias, incluida la chilena ${ }^{45,49,50}$. En negrilla se muestran las frecuencias más altas en población chilena que podrían impactar en el riesgo de desarrollar diversos tipos de cáncer asociados al consumo de tabaco o alcohol.

En la Tabla 3 se muestran las asociaciones encontradas por nuestro grupo de investigación entre la presencia de polimorfismos de enzimas fase I y fase II, el hábito tabáquico o alcohólico y el mayor riesgo de desarrollar cáncer oral, cáncer laringe, cáncer gástrico y cáncer de pulmón en población chilena ${ }^{29,46,47,48}$, lo anterior también se ha descrito en otras etnias.

\section{Conclusiones}

Los datos existentes a nivel mundial y los resultados de nuestro grupo de investigación sugieren que en las interacciones genético-ambientales, la presencia de las variantes genéticas de enzimas de biotransformación fase I (CYP1A1, CYP1E1) y fase II (GST), además de polimorfismos en el sistema de reparación de daño al ADN (ERCC1/ERCC2) tendrían un efecto modificador del riesgo de cáncer inducido por el hábito de fumar y el consumo de alcohol. Esta asociación es im- 
Tabla 3. Valores de asociación de riesgo (OR) entre la presencia de polimorfismos genéticos de enzimas fase I y fase II, hábito tabáquico o alcohólico y el mayor riesgo de desarrollar cáncer en población chilena ${ }^{29,46,47,48}$

\begin{tabular}{lccc}
\hline & OR & IC95\% & P value \\
\hline Cáncer oral & & & 0,0130 \\
CYP1A1*2A*2A/GSTM1 null + Hábito tabáquico & 21,39 & $1,92-237,68$ & 0,0030 \\
CYP1A1*1*2A/GSTM1null + Hábito tabáquico & 19,14 & $2,82-129,95$ & $<0,0001$ \\
GSTM1null + Hábito tabáquico & 20,81 & $5,45-79,34$ & 0,0420 \\
CYP1A1*2A*2A + Hábito tabáquico & 7,77 & $1,07-55,91$ & 0,1760 \\
Carcinoma de células escamosas de laringe & & & $<0,0049$ \\
CYP1A1*2A*2A + Hábito tabáquico & 9,45 & $0,59-291,48$ & 0,3802 \\
GSTM1 null + Hábito tabáquico & 7,81 & $1,63-42,04$ & $<0,05$ \\
CYP1A1*2A*2A/GSTM1null + Hábito tabáquico & 16,0 & $0,0-1089,68$ & $<0,05$ \\
Cáncer Gástrico & & & $<0,05$ \\
CYP1A1*2A*2A + Hábito tabáquico & 8,37 & $1,86-37,64$ & $<0,05$ \\
GSTM1 null + Hábito tabáquico & 2,40 & $1,00-5,78$ & $3,15-59,05$ \\
CYP1A1*2A*2A + Hábito alcohólico & 13,68 & $1,23-9,16$ & 0,0010 \\
GSTM1 null + Hábito alcohólico & 3,36 & & 0,0300 \\
Cáncer pulmón & & $1,78-10,82$ & 0,0003 \\
CYP1A1*2A*2A/*1*2A + Hábito tabáquico & 4,37 & $1,54-8,37$ & 0,0017 \\
CYP1A1*2C*2C/*1*2C + Hábito tabáquico & 4,05 & $1,53-13,29$ & \\
GSTM1 null + Hábito tabáquico & 3,47 & 3,68 & \\
CYP1A1*2A*2A/*1*2A + GSTM1 null + Hábito tabáquico & & \\
\hline
\end{tabular}

portante, si consideramos que en la población chilena el hábito de fumar y beber alcohol es muy prevalente $(33,6 \%$ y $79,1 \%$, respectivamente). Finalmente, las correlaciones entre variantes genéticas de enzimas de biotransformación y sistemas de reparación del ADN, exposición medioambiental y susceptibilidad a cáncer constituyen un área prometedora de investigación básico-clínica que permitirá una mejor comprensión de por qué individuos con un grupo de genes casi idénticos pueden ser variables en la generación de numerosas patologías, en particular del cáncer.

\section{Conflicto de intereses}

Los autores declaran no tener conflicto de intereses.

\section{Agradecimientos}

Los autores del presente trabajo desean agradecer el soporte económico brindado a su línea de investigación por CONICYT a través del proyecto Fondecyt 1140434.

\section{Referencias}

1. OMS. Cancer Fact Sheets 01 febrero de 2018 [consulta el 10 de mayo de 2018]. disponible en: http://www.who. int/es/news-room/fact-sheets/detail/ cancer

2. OMS Cáncer Infantil [consulta el 9 de mayo de 2018] disponible en: http://www. who.int/cancer/media/news/Childhood_ cancer_day/es/

3. Estrategia Nacional de Cáncer. Chile 2016. [consulta el 10 de mayo de 2018].
Disponible en: http://www.minsal.cl/ wp-content/uploads/2016/10/EstrategiaNacional-de-Cancer-version-consultapublica.pdf

4. DEIS. 2018 Serie Defunciones y Mortalidad por Tumores Malignos según Sexo, Chile 1997-2013 [consulta el 3 de mayo de 2018]. Disponible en: www.deis. $\mathrm{cl}$

5. Globocan 2012. Estimated agestandardized rates (World) of deaths, worldwide (top 10 cancer sites) in 2012 [consulta el 03 de mayo de 2018].
Disponible en: http://globocan.iarc.fr/ Default.aspx

6. SENDA 2018. Estudio Nacional de Drogas en Población Escolar 2015 [consulta el 16 de mayo de 2018] Disponible en: http:// www.senda.gob.cl/observatorio/estudios/ enpe-2015/

7. Encuesta Nacional de Salud de Chile 2016-2017 [consulta el 3 de mayo de 2018] Disponible en: http://www.minsal. cl/wp-content/uploads/2017/11/ENS2016-17_PRIMEROS-RESULTADOS. pdf 
8. Jóvenes y Tabaco en las Américas Resultados de la Encuesta Mundial de Tabaquismo En Jóvenes 20002010 [consulta el 3 de mayo de 2018] Disponible en: http://www.paho. org/hq/index.php?option= com $_{-}$ docman\&task=doc_view\&gid $=24893 \&$ Ite mid=270\&lang=en

9. Lightwood JM, Glantz SA. Short-Term Economic and Health Benefits of Smoking Cessation: Myocardial Infarction and Stroke. Circulation. 1997;96(4):108996.

10. Jemal A, Thun MJ, Ries LA et al. Report to the Nation on the Status of Cancer, 1975-2005 [Internet]. Rockville MD, National Cancer Institute; 2008 [consulta el 7 de febrero de 2013]. Disponible en: http://seer.cancer.gov/report_to_nation/ archive.html

11. Pichón Riviere A, Bardach A, Caporale J, et al. Carga de Enfermedad atribuible al Tabaquismo en Chile. Documento Técnico IECS $\mathrm{N}^{\circ} 8$. Instituto de Efectividad Clínica y Sanitaria, Buenos Aires, Argentina [consulta el 3 de mayo de 2018] Disponible en: https:// www.iecs.org.ar/wp-content/uploads/ tabaquismo23-05-2014Chi-FINAL-1.pdf

12. Pallis AG, Syrigos KN. Lung cancer in never smokers: disease characteristics and risk factors. Crit Rev Oncol Hematol 2013;88(3):494-503.

13. Ramírez N, Özel MZ, Lewis AC, Marcé RM, Borrull F, Hamilton JF. Exposure to nitrosamines in thirdhand tobacco smoke increases cancer risk in non-smokers. Environ Int 2014;71:139-47.

14. Huang Y, Huang J, Lan H, Zhao GY, Huang CZ. A meta-analysis of parental smoking and the risk of childhood brain tumors. PLoS One 2014;9(7):e102910.

15. OMS. Alcohol facts sheet 5 febrero de 2018 [consulta el 4 de mayo de 2018] disponible en: http://www.who.int/es/ news-room/fact-sheets/detail/alcohol

16. Estudio de Carga de enfermedad y carga atribuible. Santiago: Ministerio de Salud / Pontificia Universidad Católica de Chile; 2007 [consulta el 3 de mayo de 2018]. Disponible en: http://epi.minsal.cl/epi/ html/invest/cargaenf2008/Informe $\% 20$ final\%20carga_Enf_2007.pdf

17. Encuesta Nacional de Salud de Chile 2009-2010 [consulta el 2 de mayo de 2018] Disponible en: http:// www.minsal.cl/portal/url/item/ bcb03d7bc28b64dfe040010165012d23. pdf.

18. SENDA, Décimo Segundo Estudio Nacional de Drogas en Población General, 2016 [consulta el 3 de mayo de 2018] Disponible en: http://www.senda.gob. $\mathrm{cl} /$ wp-content/uploads/media/estudios/ PG/2016_Estudio_Drogas_Poblacion_ General.pdf
19. Yin G, Morita M, Ohnaka K, et al. Genetic Polymorphisms of XRCC1, Alcohol Consumption, and the Risk of Colorectal Cancer in Japan. J Epidemiol. 2012;22:6471.

20. Coronado GD, Beasley J, Livaudais J. Alcohol consumption and the risk of breast cancer. Salud Publica Mex. 2011;53(5):440-7.

21. Parkin DM, Boyd L, Walker LC. The fraction of cancer attributable to lifestyle and environmental factors in the UK in 2010. Br J Cancer. 2011;105(52):577-81.

22. Breslow R, Chen Ch, Grabaud B, Mukamad K. Prospective study of alcohol consumption quantity and frequency cancer-specific mortality in US population. Am J Epidemiol. 2011; 174 (9): 1044-53.

23. Fedirko V, Tramacere I, Bagnardi V, et al. Alcohol drinking and colorectal cancer risk: an overall and dose-response metaanalysis on publishing studies. Ann Onco. 2011; 22: 1958-72.

24. Plichart M, Menegaux F, Lacour B, Hartmann O, Frappaz D, Doz F, et al. Parental smoking, maternal alcohol, coffee and tea consumption during pregnancy and childhood malignant central nervous system tumours: the ESCALE study (SFCE). Eur J Cancer Prev. 2008;17(4):376-83

25. Quiñones, L, Berthou F, Varela N, Simon B, Gil L, Lucas D. Ethnic susceptibility to lung cancer: differences in CYP2E1, CYP1A1 and GSTM1 genetic polymorphisms between French Caucasian and Chilean populations, Cancer Letters 1999;141:167-71.

26. Cascorbi L, Brockmoller J, Roots L. A C4887A polymorphism in exon 7 of human CYP1A1: population frequency, mutation linkages and impact on lung cancer susceptibility, Cancer Res 1996;56: 4965-69.

27. Marinković, N., Pašalić, D., Potočki, S. Polymorphisms of genes involved in polycyclic aromatic hydrocarbons' biotransformation and atherosclerosis. Biochemia Medica. 2013;23(3):255-65.

28. Nock NL, Tang D, Rundle A, et al. Associations between smoking, polymorphisms in polycyclic aromatic hydrocarbon (PAH) metabolism and conjugation genes and PAH-DNA adducts in prostate tumors differ by race. Cancer Epidemiol Biomarkers Prev. 2007; 16(6):1236-45

29. Quiñones L, Lucas D, Godoy J, et al. CYP1A1, CYP2E1 and GSTM1 genetic polymorphisms. The effect of single and combined genotypes on lung cancer susceptibility in Chilean people, Cancer Letters 2001;174:35-44.

30. Yamaguti GG, Lourenco GJ, Silveira VS, et al. Increased risk for acute lymphoblastic leukemia in children with cytochrome P450A1 (CYP1A1)- and $\mathrm{NAD}(\mathrm{P}) \mathrm{H}$ : quinone oxidoreductase 1 (NQO1)-inherited gene variants. Acta Haematol. 2010; 124:182-4.

31. Singhal SS, Singh SP, Singhal P, Horne D, Singhal J, Awasthi S. Antioxidant Role of Glutathione S-Transferases: 4-Hydroxynonenal, a Key Molecule in Stress-Mediated Signaling. Toxicology and applied pharmacology. 2015;289(3):361-70.

32. Schärer OD. Nucleotide Excision Repair in Eukaryotes. Cold Spring Harb Perspect Biol 2013; 5: a012609.

33. Lunn RM, Helzlsouer KJ, Parshad R, et al. XPD polymorphisms: effects on DNA repair proficiency. Carcinogenesis 2000; 21:551-5.

34. Zhang L, Wang J, Xu L, et al. Nucleotide excision repair gene ERCC1 polymorphisms contribute to cancer susceptibility: a meta-analysis. Mutagenesis 2012;27(1):67-76.

35. Benhamou S, Sarasin A. ERCC2/XPD gene polymorphisms and cancer risk. Mutagenesis. 2002;17(6):463-9.

36. Paszkowska-Szczur K, Scott RJ, Górski B, et al. Polymorphisms in nucleotide excision repair genes and susceptibility to colorectal cancer in the Polish population. Molecular Biology Reports 2015;42(3):755-64.

37. Zhao R, Ying MF. Association between ERCC1 and ERCC2 polymorphisms and breast cancer risk in a Chinese population. Genet Mol Res. 2016;15(1):15017263.

38. Guo XF, Wang J, Lei XF, et al. XPD Asp312Asn polymorphism and esophageal cancer risk: an update metaanalysis based on 3928 cases and 6012 controls. Int J Clin Exp Med. 2014;7:244352.

39. Xue H, Lu Y, Lin B, Chen J, Tang F, Huan GG. The effect of XPD/ERCC2 polymorphisms on gastric cancer risk among different ethnicities: A systematic review and meta-analysis. PloS one. 2012;7(9):e43431.

40. Bănescu C, Trifa AP, Demian S, et al. Polymorphism of XRCC1, XRCC3, and XPD genes and risk of chronic myeloid leukemia. Biomed Res Int. 2014(4):213790.

41. Cao Y, Giovannucci EL. Alcohol as a risk factor for cancer. Seminars in Oncology Nursing. 2016;32(3):325-31.

42. Seitz H, Becker P. Alcohol Metabolism and cancer risk. Alcohol Research and Health. 2007;30(1):38-47.

43. Bonola IF, Irigoyen ME, Vera0 LI, Campero A, Hamdan A. Estrés oxidante: el sistema enzimático glutatión y la salud bucal. Ciencias Clínicas. 2014;15(1):2-8.

44. Wang Y, He J, Ma T-J, et al. GSTT1 null genotype significantly increases the 
susceptibility to urinary system cancer: Evidences from 63.876 subjects. Journal of Cancer. 2016;7:1680-93.

45. Roco AM, Quiñones LA, Agúndez JA, et al. "Frequencies of 23 functionally significant variant alleles related with metabolism of antineoplastic drugs in the Chilean population: comparison with Caucasian and Asian populations". Front Genet. 2012;3:229.

46. Cordero K, Espinoza I, Caceres D, et al. "Oral cancer susceptibility associated with the CYP1A1 and GSTM1 genotypes in Chilean individuals". Oncology Letters 2010;1(3):549-53.
47. Rahal M, Herrera MJ, Quiñones L, Farfan N, Cáceres D, Roco A. "Frecuencia de los polimorfismos CYP1A1*2A y deleción del gen GSTM1 en pacientes con carcinoma de células escamosas de laringe en relación al hábito tabáquico: Estudio piloto en Chile” Rev. Otorrinolaringol. Cir. Cabeza Cuello. 2013;73:7-16.

48. Kuen L, Cáceres D, Varela N, Csendes A, Ríos H, Quiñones L. Variantes alélicas de CYP1A1 y GSTM1 como biomarcadores de susceptibilidad a cáncer gástrico: influencia de los hábitos tabáquico y alcohólico. Rev Med Chile. 2006;134(9):1107-15.
49. Roco A. Factores genéticos relacionados con la variabilidad en la respuesta a la quimioterapia en pacientes con cáncer testicular. Tesis Programa de Doctorado en Biomarcadores de Salud y Estados Patológicos por la Universidad de Extremadura. 2016. [consulta el 2 de mayo de 2018] Disponible en: https://www.educacion.gob.es/ teseo/mostrarRef.do?ref $=1619802$.

50. Proyecto 1000 genomas. Consultada el 1 de mayo de 2018. Disponible en: http://www.ensembl.org/Homo_sapiens/ Info/Index 